\title{
Perioperative Supplemental Oxygen and Plasma Catecholamine Concentrations After Major Abdominal Surgery - a Substudy of a Randomized Clinical Trial
}

\author{
Alexander Taschner \\ Medical University of Vienna https://orcid.org/0000-0001-7305-9941 \\ Barbara Kabon \\ Medical University of Vienna \\ Markus Falkner von Sonnenburg \\ Medical University of Vienna \\ Alexandra Graf \\ Medical University of Vienna \\ Nikolas Adamowitsch \\ Medical University of Vienna \\ Melanie Fraunschiel \\ Medical University of Vienna \\ Edith Fleischmann \\ Medical University of Vienna \\ Christian Reiterer ( $\nabla$ christian.reiterer@meduniwien.ac.at ) \\ Medical University of Vienna
}

\section{Research}

Keywords: Perioperative Medicine, Cardiovascular Risk, Major abdominal surgery, MINS, Catecholamines, Hyperoxia

Posted Date: October 13th, 2021

DOI: https://doi.org/10.21203/rs.3.rs-958955/v1

License: (1) This work is licensed under a Creative Commons Attribution 4.0 International License. Read Full License 


\section{Abstract}

\section{Background}

Increased sympathetic nerve activity due to perioperative stress is associated with higher plasma catecholamine concentrations that lead to an increase in heart rate and blood pressure. This fact plays a pivotal role in the development of perioperative myocardial ischemia. A previous study in healthy volunteers has shown that the administration of supplemental oxygen attenuated sympathetic nerve activity, which was associated with lower plasma catecholamine concentrations. However, in patients undergoing surgery evidence is still lacking. We therefore tested the hypothesis that perioperative supplemental oxygen attenuates sympathetic nerve activity estimated by plasma catecholamines in patients at risk for cardiovascular complications undergoing major abdominal surgery.

Methods

We randomly assigned 81 patients to receive either $80 \%$ versus $30 \%$ inspired oxygen concentration throughout surgery and for the first two postoperative hours. We assessed noradrenaline, adrenaline and dopamine plasma concentrations as surrogate parameters for sympathetic nerve activity before induction of anesthesia, two hours after surgery and on the third postoperative day.

Results

41 patients received $80 \%$ oxygen and 40 patients received $30 \%$ oxygen. There was no significant difference in postoperative noradrenaline (effect estimated:-41.5 ng. $\mathrm{L}^{-1}, 95 \% \mathrm{Cl}-134.3,51.2 ; \mathrm{p}=0.38$ ), adrenaline (effect estimated:11.2 $\mathrm{ng} . \mathrm{L}^{-1}, 95 \% \mathrm{Cl}-7.6,30.1 ; \mathrm{p}=0.24$ ) and dopamine (effect estimated:-1.61 ng. $\left.\mathrm{L}^{-1}, 95 \% \mathrm{Cl}-7.2,3.9 ; \mathrm{p}=0.57\right)$ concentrations between both groups.

\section{Conclusions}

We found no significant difference in postoperative plasma catecholamine concentration between the $80 \%$ and $30 \%$ oxygen group in patients at risk for cardiovascular complications undergoing major abdominal surgery. Based on our results, it seems likely that supplemental oxygen did not influence sympathetic nerve activity in the perioperative setting.

Trial Registration

ClinicalTrials.gov (NCT 03366857)

European Clinical Trial Database (EudraCT 2017-003714-68)

\section{Background}


Surgery is associated with an increased stress response that triggers sympathetic nerve activity, leading to an increased release of endogenous plasma catecholamines. ${ }^{1,2}$ This causes a significant increase in heart rate and blood pressure, which has been shown to be associated with a higher risk of developing myocardial injury after non-cardiac surgery (MINS) ${ }^{2-4}$ Moreover, in the non-operating setting elevated endogenous plasma catecholamine levels are associated with the development and progression of cardiac related diseases. ${ }^{5,6}$

A large randomized trial has shown that perioperative sympathetic nerve blockade with metoprolol significantly decreased the incidence of postoperative myocardial infarction in patients undergoing noncardiac surgery. ${ }^{3}$ The authors suggested that a decrease of heart rate and blood pressure is associated with a simultaneous decrease in myocardial oxygen consumption that consequently resulted in a lower incidence of myocardial perfusion related complications. ${ }^{3}$ Additionally, higher plasma catecholamine levels stimulate platelet aggregation, which is another trigger factor for the development of acute coronary stenosis. $2,7,8$ Moreover, another study has shown that hypertension causes endovascular shear stress that might further be a contributing factor for the development and progression of myocardial ischemia. ${ }^{2}$

Interestingly, in the non-surgical setting the administration of supplemental oxygen decreases sympathetic nerve activity, which was determined by measuring plasma noradrenaline and adrenaline concentrations. ${ }^{9,10}$ This effect has been observed in patients with central sleep apnea and has been explained by a reduction in hypoxic episodes, which are common in patients with sleep apnea. ${ }^{9}$ However, these results are based on a small number of patients and data from the perioperative setting are still missing. ${ }^{9,10}$

Higher oxygen concentrations are still commonly administered during surgery and recommended by the WHO to reduce the risk of postoperative wound infections. ${ }^{11}$ Therefore, it is still of clinical interest if the perioperative administration of supplemental oxygen also attenuates the effect of sympathetic nerve activity, specifically in patients at-risk for cardiovascular complications undergoing major abdominal surgery.

In this context, we tested the hypothesis that the perioperative administration of $80 \%$ oxygen leads to a significant decrease in postoperative sympathetic nerve activity, which was assessed with consecutive plasma adrenaline, noradrenaline and dopamine concentration measurements, as compared to the administration of $30 \%$ oxygen in patients at-risk for cardiovascular complications undergoing major abdominal surgery. Moreover, to evaluate the association between plasma catecholamine concentrations and the incidence of MINS we compared the plasma catecholamine concentrations between patients, who developed MINS and patients, who did not develop MINS in the first three postoperative days.

\section{Methods}




\section{Study Design and Participants}

This is a pre-planned sub-study of a single-center, double-blinded, randomized clinical trial, which investigated the effect of supplemental oxygen on postoperative maximum NT-pro-BNP concentrations in patients at-risk for cardiovascular complications undergoing major abdominal surgery. The trial was conducted at the Medical University of Vienna. ${ }^{12}$ This sub-study and the original trial were approved by the local Institutional Review Board and were registered at ClinicalTrials.gov (NCT 03366857) and at the European Clinical Trial Database (EudraCT 2017-003714-68). The study protocol was published previously. ${ }^{13}$

We obtained written informed consent from all patients before randomization. We included 82 consecutive patients enrolled into the main study and scheduled for major abdominal surgery expected to last at least 2 hours. Eligible patients were over 45 years and underwent noncardiac surgery under general anesthesia. Patients had to meet one or more of the following criteria: 1 . History of coronary artery disease; 2. History of peripheral arterial disease; 3 . History of stroke or 4 . Any three of the following six criteria (a-f): a) age over 70 years; b) undergoing major surgery; c) history of congestive heart failure; d) history of transient ischemic attack; e) diabetes and currently taking an oral hypoglycemic agent or insulin; f) history of hypertension. We excluded patients with one of the following criteria: 1 . septic patients, 2. preoperative inotropic therapy, 3. oxygen dependent patients, or 4 . history of severe heart failure (defined as ejection fraction $<30 \%$ ).

\section{Randomization}

We randomized patients using a web-based randomization program (Randomizer, Medical University of Graz, Graz, Austria, https://www.meduniwien.ac.at/randomizer/web). Randomization sequence was generated by the study statistician using permutated blocks. Each block had a size of 6 numbers, of which all investigators were unaware. There was no stratification of randomization.

Shortly before induction of anesthesia, we randomized patients to receive either $80 \%$ or $30 \%$ inspired oxygen concentration throughout surgery, and for two hours postoperatively. After endotracheal intubation patients in the $80 \%$ oxygen group received an inspired oxygen fraction of 0.8 throughout surgery and $8 \mathrm{~L} / \mathrm{min}$ oxygen via facemask with reservoir for the first two postoperative hours. Patients in the $30 \%$ oxygen group received an inspired oxygen fraction of 0.3 throughout surgery and $3 \mathrm{~L} / \mathrm{min}$ oxygen via facemask without a reservoir for the first two postoperative hours. If needed, oxygen fraction was increased at the discretion of the attending anesthetist according to a predefined algorithm. ${ }^{13}$

\section{Protocol}

We administered 1-3 $\mathrm{g} \mathrm{kg}^{-1}$ body weight (BW) fentanyl, $2 \mathrm{mg} \mathrm{kg}^{-1} \mathrm{BW}$ propofol and $0.6 \mathrm{mg} \mathrm{kg}^{-1}$ rocuronium for induction of anesthesia. All patients had EKG, blood pressure and $\mathrm{SpO}_{2}$ monitoring. After induction of anesthesia, continuous invasive arterial blood pressure measurements were initiated. A central venous line was inserted at the discretion of the attending anesthesiologist. 
Anesthesia was maintained with sevoflurane (up to $1.5 \mathrm{MAC}$ ) according to processed EEG-guided anesthesia. The randomly assigned oxygen concentration was set immediately after endotracheal intubation and was maintained throughout surgery. During induction of anesthesia and during extubation we used $100 \%$ oxygen to meet the local safety standards.

Muscle relaxation was given when necessary to maintain 1-2 mechanical twitches in response to supramaximal stimulation (Train-of-Four stimulation, target $<75 \%$ ). Core temperature was maintained above $36^{\circ} \mathrm{C}$ using forced-air warming.

All patients received $2-3 \mathrm{~mL} / \mathrm{kg}$ BW baseline balanced crystalloid infusion. We administered a bolus of $250 \mathrm{~mL}$ of balanced crystalloid solution guided by esophageal Doppler (Cardia Q, Deltex Chichester, UK) according to a previous published algorithm. ${ }^{14,15}$ In the case of intraoperative hypotension (mean blood pressure $<65 \mathrm{mmHg}$ ) either fluids or vasopressors were given as necessary to maintain hemodynamic stability. Colloids, blood and blood products were given per clinical judgement.

\section{Measurements}

We recorded demographic data including age, sex, BMI, American Society of Anaesthesiologists (ASA) physical status, comorbidities, long-term medication, type of surgery and preoperative laboratory values. We also recorded routine intraoperative variables including duration of anesthesia and surgery, fluid and anesthesia management, and hemodynamic parameters and blood gas analysis. We performed blood gas analysis hourly. Blood pressure and oxygen saturation were recorded intraoperatively and for the first two postoperative hours. Intraoperative core temperature was measured at the distal esophagus. We also recorded the amount of piritramide and fluids administered during the first three postoperative days on the ward.

Blinded research personnel drew all study specific pre and postoperative blood samples. In all patients, noradrenaline, adrenaline and dopamine plasma concentrations were assessed shortly before induction of anesthesia, within two hours after the end of surgery and on the third postoperative day. Troponin $T$ concentrations for MINS diagnosis was measured shortly before induction of anesthesia, within two hours after the end of surgery, on the first and on the third postoperative day.

All laboratory measurements were performed by the department for laboratory medicine at the Medical University of Vienna.

\section{Data management}

Blinded research personnel obtained all postoperative data. All data were recorded and stored in the data management system 'Clincase', v2.7.0.12 hosted by IT Systems \& Communications, Medical University of Vienna, Vienna, Austria.

\section{Statistical Analysis}


We performed intention-to-treat analysis according to the allocated randomization. Statistical analyses were performed with SPSS (Version 26, IBM SPSS Statistic, Armonk, NY, USA). Continuous variables were summarized using mean, standard deviation (SD), median, quartiles [25th percentile; 75th percentile] as well as minimum and maximum values. Descriptive statistics are given for randomized groups separately. Categorical variables were summarized using absolute and percent values.

\section{Plasma catecholamines}

For each plasma catecholamine we performed a repeated measure mixed linear model to calculate the estimates and confidence intervals for the effect of $80 \%$ versus $30 \%$ oxygen concentration on postoperative noradrenaline, adrenaline and dopamine plasma concentrations. Oxygen concentrations were defined as fixed effects. Furthermore, values per time point were compared between groups using two-tailed Mann-Whitney U-Tests.

\section{Post-hoc analysis}

To evaluate, if patients with MINS had higher stress levels represented by significantly increased plasma catecholamines concentrations, we further stratified our patients into patients with MINS and no-MINS. Therefore, we evaluated differences in maximum plasma catecholamine concentrations between MINS and no-MINS using a Mann-Whitney-U test. Maximum plasma catecholamine concentrations were used to reflect the impact of MINS on the stress response. MINS was defined as an elevated postoperative high sensitivity Troponin T concentration of $20-65 \mathrm{ng} / \mathrm{L}$ with an absolute change of at least $5 \mathrm{ng} / \mathrm{L}$ from preoperatively or a concentration exceeding $65 \mathrm{ng} / \mathrm{L}$ regardless of the baseline value, in the absence of nonischemic causes (sepsis, atrial fibrillation, pulmonary embolism). ${ }^{16}$

All $p$-values $<0.05$ were considered as statistically significant.

\section{Sample Size}

Since this is a sub-study, the planning of the original sample size was based on the original doubleblinded, randomized clinical trial, which investigated the effect of supplemental oxygen on postoperative maximum NT-pro-BNP. ${ }^{12}$ However, we additionally estimated the number of patients required for this substudy based on a previous study that evaluated the effect of surgery on postoperative plasma catecholamine concentrations. ${ }^{1}$ The study showed that postoperative plasma noradrenaline increased on the second postoperative day to $676 \mathrm{ng} . \mathrm{L}^{-1}\left( \pm 210 \mathrm{ng. \textrm {L } ^ { - 1 }}\right)$ as compared to preoperative baseline values. ${ }^{1}$ We assumed a similar postoperative increase in our $30 \%$ oxygen group and anticipated a clinically meaningful lower increase of $20 \%$ in our $80 \%$ oxygen group. Thus, we calculated that at least 39 patients per group have $80 \%$ power to detect a significant difference at an alpha of 0.05 . To compensate for potential dropouts, we included 41 patients per group.

\section{Results}


We enrolled 82 patients undergoing major abdominal surgery at-risk for cardiovascular complications from December 2017 to July 2018. One patient in the 30\% oxygen group was excluded after randomization because surgery was postponed. Overall, 41 patients were randomly assigned to receive $80 \%$ inspired oxygen concentration and 40 patients to receive $30 \%$ inspired oxygen concentration throughout surgery and for two hours postoperatively.

Patient characteristics, ASA physical status, comorbidities, long-term medication, type of surgery and baseline laboratory parameters were similar between both groups (Table 1). Similarly, intraoperative and postoperative variables such as duration of anesthesia and surgery, fluid management, anesthesia management, hemodynamic parameters and arterial blood gas analysis were balanced between both groups. The number of patients requiring intraoperative vasopressors, as well as the overall amount of vasopressors administered was similar between both study groups (Table 2). Postoperative heart rate and blood pressure did not differ between the groups. There was also no significant difference in fluid and opioid administration within the first three postoperative days (Table 2). 
Table 1

Patient characteristics

\begin{tabular}{|c|c|c|c|c|}
\hline \multirow[b]{2}{*}{ Age, yrs } & \multicolumn{2}{|c|}{$\begin{array}{l}80 \% \text { Oxygen } \\
(n=41)\end{array}$} & \multicolumn{2}{|c|}{$\begin{array}{l}30 \% \text { Oxygen } \\
(n=40)\end{array}$} \\
\hline & 75 & {$[70 ; 78]$} & 73 & {$[69 ; 77]$} \\
\hline Height, $\mathrm{cm}$ & 170 & {$[167 ; 175]$} & 174 & {$[168 ; 179]$} \\
\hline Weight, $k g$ & 80 & {$[72 ; 88]$} & 84 & {$[77 ; 92]$} \\
\hline $\mathrm{BMI}, \mathrm{kg} \cdot \mathrm{m}^{-2}$ & 26.8 & {$[24.1 ; 29.8]$} & 27.6 & {$[25.2 ; 29.9]$} \\
\hline \multicolumn{5}{|l|}{$\operatorname{Sex}, n(\%)$} \\
\hline Women & 15 & $(36.7)$ & 9 & $(22.5)$ \\
\hline Men & 26 & $(63.3)$ & 31 & $(77.5)$ \\
\hline \multicolumn{5}{|l|}{ ASA physical status, $n(\%)$} \\
\hline II & 13 & $(36.6)$ & 16 & $(40)$ \\
\hline III & 28 & $(63.4)$ & 24 & $(60)$ \\
\hline \multicolumn{5}{|l|}{ Comorbidities, $n$ (\%) } \\
\hline Hypertension & 39 & $(95.1)$ & 36 & $(90.0)$ \\
\hline Coronary artery disease & 9 & $(21.9)$ & 8 & $(20.0)$ \\
\hline Peripheral artery disease & 6 & $(14.6)$ & 5 & $(12.5)$ \\
\hline Stroke & 5 & $(12.2)$ & 4 & $(10.0)$ \\
\hline Congestive heart failure & 3 & $(7.3)$ & 3 & $(7.5)$ \\
\hline Transient ischemic attack & 1 & $(2.4)$ & 6 & $(15.0)$ \\
\hline Insulin use & 13 & $(31.7)$ & 12 & $(30.0)$ \\
\hline \multicolumn{5}{|l|}{ Long-term medication, $n$ (\%) } \\
\hline Beta blockers & 17 & $(41.5)$ & 16 & $(40.0)$ \\
\hline $\mathrm{ACl} / \mathrm{ARB}$ & 24 & $(58.5)$ & 21 & $(52.5)$ \\
\hline Diuretics & 12 & $(29.3)$ & 6 & $(15.0)$ \\
\hline Statins & 20 & $(48.8)$ & 18 & $(45.0)$ \\
\hline Acetylsalicylic acid & 2 & $(4.9)$ & 3 & $(7.5)$ \\
\hline Oral anticoagulant & 18 & $(43.9)$ & 15 & $(37.5)$ \\
\hline Alpha 2 agonist & 1 & $(2.4)$ & 2 & $(5.0)$ \\
\hline
\end{tabular}




\begin{tabular}{|c|c|c|c|c|}
\hline & \multicolumn{2}{|c|}{$\begin{array}{l}\text { 80\% Oxygen } \\
(n=41)\end{array}$} & \multicolumn{2}{|c|}{$\begin{array}{l}30 \% \text { Oxygen } \\
(n=40)\end{array}$} \\
\hline Hepatobiliary & 8 & $(19.5)$ & 7 & $(17.5)$ \\
\hline Colorectal & 9 & $(22.0)$ & 8 & $(20.0)$ \\
\hline Pancreatic & 6 & $(14.6)$ & 3 & $(7.5)$ \\
\hline Urological & 12 & $(29.3)$ & 19 & $(47.5)$ \\
\hline Other & 6 & $(14.6)$ & 3 & $(7.5)$ \\
\hline \multicolumn{5}{|l|}{ Laboratory parameters } \\
\hline CRP, $m g . d L^{-1}$ & 0.33 & {$[0.19 ; 1.43]$} & 0.26 & {$[0.12 ; 0.58]$} \\
\hline Creatinine, $m g \cdot d L^{-1}$ & 0.9 & {$[0.8 ; 1.0]$} & 0.9 & {$[0.8 ; 1.1]$} \\
\hline Leukocytes, G.L $L^{-1}$ & 6.61 & {$[5.42 ; 8.60]$} & 6.58 & {$[5.03 ; 8.07]$} \\
\hline NT-proBNP, pg.m/ ${ }^{-1}$ & 280 & {$[97 ; 533]$} & 128 & {$[69 ; 391]$} \\
\hline Troponin T, ng. $L^{-1}$ & 14 & {$[11 ; 22]$} & 15 & {$[9 ; 22]$} \\
\hline
\end{tabular}


Table 2

Perioperative Variables

$$
\begin{array}{ll}
80 \% \text { Oxygen } & 30 \% \text { Oxygen } \\
(n=41) & (n=40)
\end{array}
$$

Intraoperative

\begin{tabular}{llllll} 
Duration of anesthesia, $\min$ & 264 & {$[191 ; 403]$} & 215 & {$[177 ; 287]$} & 0.06 \\
\hline Duration of surgery, $\min$ & 207 & {$[134 ; 329]$} & 152 & {$[129 ; 233]$} & 0.17
\end{tabular}

Fluid management

Crystalloid, $m L$

$2237 \quad[1262 ; 3538] \quad 1936 \quad[1396 ; 2696] \quad 0.41$

Blood loss, $m L$

200

$[0 ; 500]$

$200 \quad[0 ; 500]$

0.78

Urine output, $m L$

$245 \quad[150 ; 400]$

$225 \quad[285 ; 400]$

0.83

Anesthesia management

Fentanyl, $m c g$

$1100 \quad[863 ; 1488] \quad 1050 \quad[800 ; 1500] \quad 0.60$

Propofol, $m g$

$145 \quad[70 ; 160]$

150 [100; 200]

0.15

Phenylephrine, $m g$

0.25

$[0.10 ; 0.58]$

$0.20 \quad[0.08 ; 0.52]$

0.56

Noradrenaline, $m g$

0.0

$[0.0 ; 1.0]$

0.0

$[0.0 ; 0.3]$

0.10

etSevo, \%

1.3

[1.0; 1.5]

1.2

$[0.7 ; 1.4]$

0.18

$\mathrm{FiO}_{2}, \%$

81

[80; 81]

32

$[31 ; 68]$

etCO ${ }_{2}, \mathrm{mmHg}$

35

Core temp, $C^{\circ}$

36.2

[34; 36]

34

$[32 ; 36]$

0.18

Core temp,

Hemodynamic Parameters

$\mathrm{HR}$, beats. $\mathrm{min}^{-1}$

62

[58; 69]

73

$[62 ; 84]$

0.59

MAP, $m m H g$

79

[74; 90]

91

[85; 95]

0.42

$S V, m L$

77

[55; 81]

52

[30;67]

0.22

CO, L. $\min ^{-1}$

4.7

[3.7; 5.4]

3.3

$[1.9 ; 4.5]$

0.12

CVP, $\mathrm{mmHg}$

$12 \quad[8 ; 15]$

11

[8; 20]

0.69

Arterial Blood Gas Analysis

$\mathrm{pO}_{2}, \mathrm{mmHg}$

$158 \quad$ [112; 195]

91

[75; 155]

$<0.05$

$\mathrm{pCO}_{2}, \mathrm{mmHg}$

43

43

[39; 52]

49

[46; 60]

0.50 


\begin{tabular}{|c|c|c|c|c|c|}
\hline \multirow[b]{2}{*}{$\mathrm{pH}$} & \multicolumn{2}{|c|}{$\begin{array}{l}80 \% \text { Oxygen } \\
(n=41)\end{array}$} & \multicolumn{2}{|c|}{$\begin{array}{l}30 \% \text { Oxygen } \\
(n=40)\end{array}$} & \multirow{2}{*}{$\begin{array}{l}\boldsymbol{p} \text {-Value } \\
0.33\end{array}$} \\
\hline & 7.34 & {$[7.28 ; 7.38]$} & 7.31 & {$[7.25 ; 7.34]$} & \\
\hline $\mathrm{BE}$ & -1.9 & {$[-4.6 ;-0.7]$} & -1.2 & {$[-3.3 ;-0.1]$} & 0.25 \\
\hline $\mathrm{Hb}, g \cdot d L^{-1}$ & 11.8 & {$[10.4 ; 12.5]$} & 13.4 & {$[11.1 ; 13.9]$} & 0.31 \\
\hline Lactate, $m m o l . L^{-1}$ & 0.8 & {$[0.6 ; 1.0]$} & 1.6 & {$[0.9 ; 2.3]$} & 0.53 \\
\hline Glucose, $m g \cdot d L^{-1}$ & 163 & {$[150 ; 184]$} & 155 & {$[123 ; 175]$} & 0.31 \\
\hline \multicolumn{6}{|l|}{2 hours postoperative } \\
\hline \multicolumn{6}{|c|}{ Hemodynamic Parameters } \\
\hline $\mathrm{HR}$, beats ${ }^{\star} \min ^{-1}$ & 75 & {$[60 ; 88]$} & 82 & {$[64 ; 90]$} & 0.41 \\
\hline MAP, $m m H g$ & 86 & {$[75 ; 103]$} & 109 & {$[98 ; 122]$} & $<0.001$ \\
\hline \multicolumn{6}{|l|}{72 hours postoperative } \\
\hline Fluid, $m L^{\text {a) }}$ & 10400 & {$[7417 ; 12525]$} & 9471 & {$[7013 ; 11526]$} & 0.58 \\
\hline Piritramide, $m g^{b)}$ & 12.0 & {$[4.5 ; 22.0]$} & 8.3 & {$[3.0 ; 21.8]$} & 0.65 \\
\hline
\end{tabular}

\section{Plasma catecholamine concentrations}

The administration of supplemental oxygen did not result in a significant difference in the postoperative plasma noradrenaline (effect estimated: $-41.5 \mathrm{ng} . \mathrm{L}^{-1}, 95 \% \mathrm{Cl}-134.3,51.2 ; p=0.38$ ), adrenaline (effect estimated: $11.2 \mathrm{ng} . \mathrm{L}^{-1}, 95 \% \mathrm{Cl}-7.6,30.1 ; p=0.24$ ) and dopamine (effect estimated: $-1.61 \mathrm{ng} . \mathrm{L}^{-1}, 95 \% \mathrm{Cl}$ $-7.2,3.9 ; p=0.57$ ) concentrations (Figure $2 \mathrm{a}-\mathrm{c}$ ) between the $80 \%$ and $30 \%$ oxygen groups within the first three postoperative days. Plasma catecholamine concentrations measured at each time point are shown in Table 3. 
Table 3

Plasma catecholamine concentrations

\begin{tabular}{|c|c|c|c|c|c|}
\hline & \multicolumn{2}{|c|}{$\begin{array}{l}80 \% \text { Oxygen } \\
(n=41)\end{array}$} & \multicolumn{2}{|c|}{$\begin{array}{l}30 \% \text { Oxygen } \\
(n=40)\end{array}$} & \multirow[t]{2}{*}{$p$-Value } \\
\hline \multicolumn{5}{|l|}{ Noradrenaline, $n g . L^{-1}$} & \\
\hline Baseline & 247 & {$[147 ; 443]$} & 259 & {$[170 ; 369]$} & 0.20 \\
\hline 2 hours postoperative & 757 & {$[447 ; 1240]$} & 494 & {$[318 ; 864]$} & 0.13 \\
\hline Postoperative day 3 & 560 & {$[384 ; 827]$} & 493 & {$[313 ; 730]$} & 0.89 \\
\hline \multicolumn{6}{|l|}{ Adrenaline, $n g . L^{-1}$} \\
\hline Baseline & 27 & {$[16 ; 43]$} & 25 & {$[14 ; 69]$} & 0.59 \\
\hline 2 hours postoperative & 187 & {$[70 ; 539]$} & 193 & {$[74 ; 444]$} & 0.86 \\
\hline Postoperative day 3 & 36 & {$[18 ; 49]$} & 43 & {$[23 ; 75]$} & 0.17 \\
\hline \multicolumn{6}{|l|}{ Dopamine, $n g . L^{-1}$} \\
\hline Baseline & 0 & {$[0 ; 19]$} & 0 & {$[0 ; 15]$} & 0.66 \\
\hline 2 hours postoperative & 34 & {$[19 ; 52]$} & 19 & {$[0 ; 35]$} & 0.10 \\
\hline Postoperative day 3 & 23 & {$[0 ; 37]$} & 0 & {$[0 ; 85]$} & 0.92 \\
\hline
\end{tabular}

\section{Post-hoc analysis}

$26(32.1 \%)$ of our patients developed MINS within three days after surgery. There was no significant difference in maximum postoperative concentrations of noradrenaline $(p=0.48)$, adrenaline $(p=0.72)$ and dopamine ( $p=0.94)$ between patients with MINS and patients without MINS (Table 4).

Table 4

Maximum plasma catecholamine concentrations and MINS

\begin{tabular}{|c|c|c|c|c|c|}
\hline \multirow[b]{2}{*}{ Maximum Noradrenaline, $n g \cdot L^{-1}$} & \multicolumn{2}{|c|}{$\begin{array}{l}\text { MINS } \\
(n=26)\end{array}$} & \multicolumn{2}{|c|}{$\begin{array}{l}\text { No MINS } \\
(n=55)\end{array}$} & \multirow{2}{*}{$\begin{array}{l}\boldsymbol{p} \text {-Value } \\
0.48\end{array}$} \\
\hline & 782 & {$[508 ; 1040]$} & 855 & {$[597 ; 1164]$} & \\
\hline Maximum Adrenaline, $n g \cdot L^{-1}$ & 171 & {$[113 ; 571]$} & 211 & {$[75 ; 438]$} & 0.72 \\
\hline Maximum Dopamine, $n g \cdot L^{-1}$ & 39 & {$[11 ; 68]$} & 36 & {$[17 ; 71]$} & 0.94 \\
\hline
\end{tabular}

\section{Discussion}


The perioperative administration of $80 \%$ versus $30 \%$ inspired oxygen concentration showed no significant effect on postoperative plasma noradrenaline, adrenaline and dopamine concentrations in patients at-risk for cardiovascular complications undergoing major abdominal surgery. Additionally, we also found no significant difference in postoperative noradrenaline, adrenaline and dopamine plasma concentrations between patients with and without MINS.

Evidence exists in the non-surgical setting that supplemental oxygen significantly decreased the release of plasma catecholamine concentrations. ${ }^{10}$ Specifically, in patients with chronic heart failure, the administration of two liters oxygen via a nasal cannula for four weeks significantly attenuated sympathetic nerve activity, which resulted in lower serum noradrenaline and adrenaline concentrations as compared to breathing air. ${ }^{10}$ Furthermore, it has been shown that the long-term administration of two liters oxygen during the night in patients with congestive heart failure and sleep apnea significantly decreased brain natriuretic peptides during a treatment period of four weeks. ${ }^{9}$ The authors concluded that oxygen administration reduced sympathetic nerve activity that finally attenuated the myocardial strain in these patients. ${ }^{9}$ In contrast to these studies we did not observe any effect in plasma catecholamine concentrations between both study groups. An explanation therefore might be that we investigated patients having major abdominal surgery and administered supplemental oxygen only throughout the immediate perioperative period. Furthermore, none of our patients suffered hypoxic events as it is commonly observed in patients with sleep apnoea. ${ }^{17,18}$ In this context, it might be possible that the intraoperative administration of supplemental oxygen leads to distinct physiological effects as compared to the nonsurgical setting.

A recent review has shown that the administration of supplemental oxygen has significant hemodynamic effects in healthy volunteers, septic patients and patients having cardiac surgery. ${ }^{19}$ Specifically, it has been shown that supplemental oxygen significantly decreases heart rate, stroke volume and cardiac output. ${ }^{19}$ In contrast, we did not find any significant differences in intraoperative hemodynamic parameters between both groups. This is consistent with the results of a previous trial and also of our main trial, in which no significant differences in intraoperative hemodynamic parameters were observed. ${ }^{12,20}$ An explanation therefore might be that all of our patients received general anesthesia. It is well known that anesthetics and opioids blunt sympathetic nerve activity, and therefore hemodynamic effects of oxygen might play a minor role in the surgical setting.

Over $90 \%$ of our patients had history of clinically relevant hypertension requiring medical treatment. Interestingly, it has been shown that patients with history of hypertension have significantly higher plasma catecholamine concentrations as compared to normotensive patients. ${ }^{21}$ Furthermore, approximately $40 \%$ of our patients also took $\beta$-blockers therapy. Since they inhibit the effect of endogenous catecholamines on receptors and not their release, there should be no influence on stress markers. ${ }^{3}$ Moreover, the number of patients with pre-existing hypertension and patients taking $\beta$-blockers was similar between both study groups. Therefore, it seems unlikely that hypertension and $\beta$-blocker therapy influenced postoperative plasma catecholamine concentrations and consequently our results. 
Pain and hypothermia, which are common in the perioperative period, are further trigger factors for stress and exacerbated catecholamine release. ${ }^{22-24}$ Therefore, we actively warmed our patients during surgery. During the perioperative period pain was treated according to our local clinical standard. In detail, all patients received metamizole or another non-steroid anti-inflammatory drug in the recovery room. If visual analogue pain score (VAS) was over 4 , we additionally administered piritramide. There was no difference in perioperative amounts of opioids administration between both groups.

It has previously been shown that perioperative elevated plasma catecholamine concentrations resulting from high blood pressure, relative insulin deficiency, surgical trauma and hypothermia are trigger factors for myocardial ischemia. ${ }^{23}$ In this context, it has been shown that patients undergoing major non-cardiac surgery, who had increased postoperative Troponin T levels also have significantly higher plasma noradrenaline and adrenaline concentrations. ${ }^{1}$ Thus, we also evaluated in a post-hoc analysis, if patients with MINS had higher plasma catecholamine concentrations as compared to those without MINS. In contrast to current literature, we did not observe significantly higher plasma catecholamine concentrations in patients with MINS as compared to patients without MINS. Nevertheless, this was a post-hoc analysis and we did not power this study to detect a significant difference in maximum catecholamine concentrations between patients with and without MINS and should therefore be investigated in future trials.

This study has some limitations. Firstly, we did not measure plasma catecholamine concentrations on the first and second postoperative day. Therefore, it might be possible that we have missed the maximum rise in postoperative plasma catecholamine concentrations. However, it has been shown that plasma catecholamine measurements on the third postoperative day accurately represent the maximal stress response in cardiac-risk patients undergoing noncardiac surgery. ${ }^{1}$

Some of our patients required a continuous infusion of noradrenaline to maintain mean arterial pressure (MAP) over $65 \mathrm{mmHg}$ during surgery. There was no difference between the number of patients requiring noradrenaline administration and the total amount of noradrenaline administered between both groups. Furthermore, since the plasma half-life of noradrenaline is only 2.5 minutes and none of our patients received a continuous noradrenaline infusion in the postoperative study period, we thus did not expect a significant influence on our results.

In summary, this sub-study did not show a significant effect of perioperative supplemental oxygen on postoperative plasma catecholamine concentrations in patients at-risk for cardiovascular complications undergoing major abdominal surgery. Our study period was limited to the immediate perioperative period. As we observed a significant increase in plasma catecholamine concentrations within the third postoperative day, further studies should focus on postoperative treatment options in order to attenuate sympathetic nerve activity.

\section{Declarations}


Ethics approval and consent to participate: This sub-study and the original trial were approved by the local Institutional Review Board (Ethikkommission Medizinische Universität Wien; EK-Number 1744/2017). Written informed consent was obtained from all patients prior to randomization.

Consent for publication: Not applicable

Availability of data and materials: The datasets used and analyzed during the current study are available from the corresponding author on reasonable request.

Competing interests: The authors declare that they have no competing interests.

Funding: The authors have no funding to declare.

Authors' contributions: AT: Data Evaluation, Statistical Analysis, Drafting Manuscript; BK: Study Protocol, Study Conduct, Drafting Manuscript; MS: Study Conduct; Alexandra Graf: Statistical Analysis; NA: Study Conduct; MF: Study Conduct; EF: Study Protocol, Drafting Manuscript; CR: Study Protocol, Study Conduct, Drafting Manuscript.

All authors contributed equally to the manuscript and read and approved the final version of the manuscript.

Acknowledgements: Not applicable

\section{References}

1. Sametz W, Metzler H, Gries M, et al. Perioperative catecholamine changes in cardiac risk patients. Eur J Clin Invest. 1999;29:582-587.

2. Parker SD, Breslow MJ, Frank SM, et al. Catecholamine and cortisol responses to lower extremity revascularization: correlation with outcome variables. Perioperative Ischemia Randomized Anesthesia Trial Study Group. Crit Care Med. 1995;23(12):1954-1961. doi:10.1097/00003246-199512000-00003

3. Devereaux PJ, Yang H, Yusuf S, et al. Effects of extended-release metoprolol succinate in patients undergoing non-cardiac surgery (POISE trial): A randomised controlled trial. Lancet. 2008;371(9627):1839-1847. doi:10.1016/S0140-6736(08)60601-7

4. Devereaux PJ, Szczeklik W. Myocardial injury after non-cardiac surgery: diagnosis and management. Eur Heart J. 2019:1-9. doi:10.1093/eurheartj/ehz301

5. Cohn JN, Levine B, Olivari MT, et al. Plasma norepinephrine as a Guide to Prognosis in Chronic Congestive Heart Failure. N Engl J Med. 1984;311:819-823.

6. Lamba S, Abraham WT. Alterations in adrenergic receptor signaling in heart failure. Heart Fail Rev. 2000;5(1):7-16. doi:10.1023/A:1009885822076 
7. Priebe HJ. Triggers of perioperative myocardial ischaemia and infarction. Br J Anaesth. 2004;93(1):9-20. doi:10.1093/bja/aeh147

8. Wu KK. Platelet activation mechanisms and markers in arterial thrombosis. J Intern Med. 1996;239(1):17-34. doi:10.1046/j.1365-2796.1996.331661000.x

9. Shigemitsu M, Nishio K, Kusuyama T, Itoh S, Konno N, Katagiri T. Nocturnal oxygen therapy prevents progress of congestive heart failure with central sleep apnea. Int J Cardiol. 2007;115(3):354-360. doi:10.1016/j.ijcard.2006.03.018

10. Staniforth AD, Kinnear WJM, Starling R, Hetmanski DJ, Cowley AJ. Effect of oxygen on sleep quality, cognitive function and sympathetic activity in patients with chronic heart failure and CheynStokes respiration. Eur Heart J. 1998;19(6):922-928. doi:10.1053/euhj.1997.0861

11. Allegranzi B, Zayed B, Bischoff $P$, et al. Surgical site infections 2 New $W H O$ recommendations on intraoperative and postoperative measures for surgical site infection prevention: an evidence-based global perspective. Lancet Infect Dis. 2016;16(12):e288-e303. doi:10.1016/S1473-3099(16)30402-9

12. Reiterer C, Kabon B, Taschner A, et al. Perioperative supplemental oxygen and NT-proBNP concentrations after major abdominal surgery - A prospective randomized clinical trial. J Clin Anesth. 2021;73(May). doi:10.1016/j.jclinane.2021.110379

13. Reiterer C, Kabon B, Von Sonnenburg MF, et al. The effect of supplemental oxygen on perioperative brain natriuretic peptide concentration in cardiac risk patients - A protocol for a prosprective randomized clinical trial. Trials. 2020;21(1):1-9. doi:10.1186/s13063-020-04336-9

14. Gan TJ, Soppitt A, Sc B, Maroof M. Goal-directed Intraoperative Fluid Administration Reduces Length of Hospital Stay after Major Surgery. Anesthesiology. 2002;97(4):820-826.

15. Feldheiser A, Conroy P, Bonomo T, Cox B. Development and Feasibility Study of an Algorithm for Intraoperative Goal- directed Haemodynamic Management in Noncardiac Surgery. J Int Med Res. 2012;40:1227-1241. doi:10.1177/147323001204000402

16. Devereaux PJ, Biccard BM, Sigamani A, et al. Association of postoperative high-sensitivity troponin levels with myocardial injury and 30-day mortality among patients undergoing noncardiac surgery. JAMA - J Am Med Assoc. 2017;317(16):1642-1651. doi:10.1001/jama.2017.4360

17. Kaljusto ML, Stensløkken KO, Mori T, et al. Preconditioning effects of steroids and hyperoxia on cardiac ischemia-reperfusion injury and vascular reactivity. Eur J Cardio-thoracic Surg. 2008;33(3):355363. doi:10.1016/j.ejcts.2007.12.017

18. Baharvand B, Dehaj ME, Foadaddini M, et al. Delayed Cardioprotective Effects of Hyperoxia Preconditioning Prolonged by Intermittent Exposure. J Surg Res. 2010;160(1):53-59.

doi:10.1016/j.jss.2008.12.034

Page 16/20 
19. Smit B, Smulders YM, van der Wouden JC, Oudemans-van Straaten HM, Spoelstra-de Man AME. Hemodynamic effects of acute hyperoxia: Systematic review and meta-analysis. Crit Care. 2018;22(1):110. doi:10.1186/s13054-018-1968-2

20. Greif R, Akca O, Horn E-P, Kurz A, Sessler DI. Supplemental Perioperative Oxygen to reduce the Incidence of Surgical-Wound Infection. N Engl J Med. 2000;342:161-167.

21. Bühler FR, Amann FW, Bolli P, et al. Elevated adrenaline and increased alpha-adrenoceptormediated vasoconstriction in essential hypertension. J Cardiovasc Pharmacol. 1982;4 Suppl 1:S134-8. doi:10.1097/00005344-198200041-00027

22. Møller IW, Dinesen K, Søndergård S, Knigge U, Kehlet H. Effect of patient-controlled analgesia on plasma catecholamine, cortisol and glucose concentrations after cholecystectomy. Br J Anaesth. 1988;61:160-164. doi:10.1093/bja/61.2.160

23. Devereaux PJ, Goldman L, Cook DJ, Gilbert K, Leslie K, Guyatt GH. Perioperative cardiac events in patients undergoing noncardiac surgery: A review of the magnitude of the problem, the pathophysiology of the events and methods to estimate and communicate risk. Cmaj. 2005;173(6):627-634. doi:10.1503/cmaj.050011

24. Frank SM, Higgins MS, Breslow MJ, et al. The Catecholamine, Cortisol, and Hemodynamic Responses to Mild Perioperative Hypothermia. Anesthesiology. 1995;82:83-93.

25. Schulz KF, Altman DG, Moher D. CONSORT 2010 statement: Updated guidelines for reporting parallel group randomised trials. Int J Surg. 2011;9(8):672-677. doi:10.1016/j.ijsu.2011.09.004

\section{Figures}




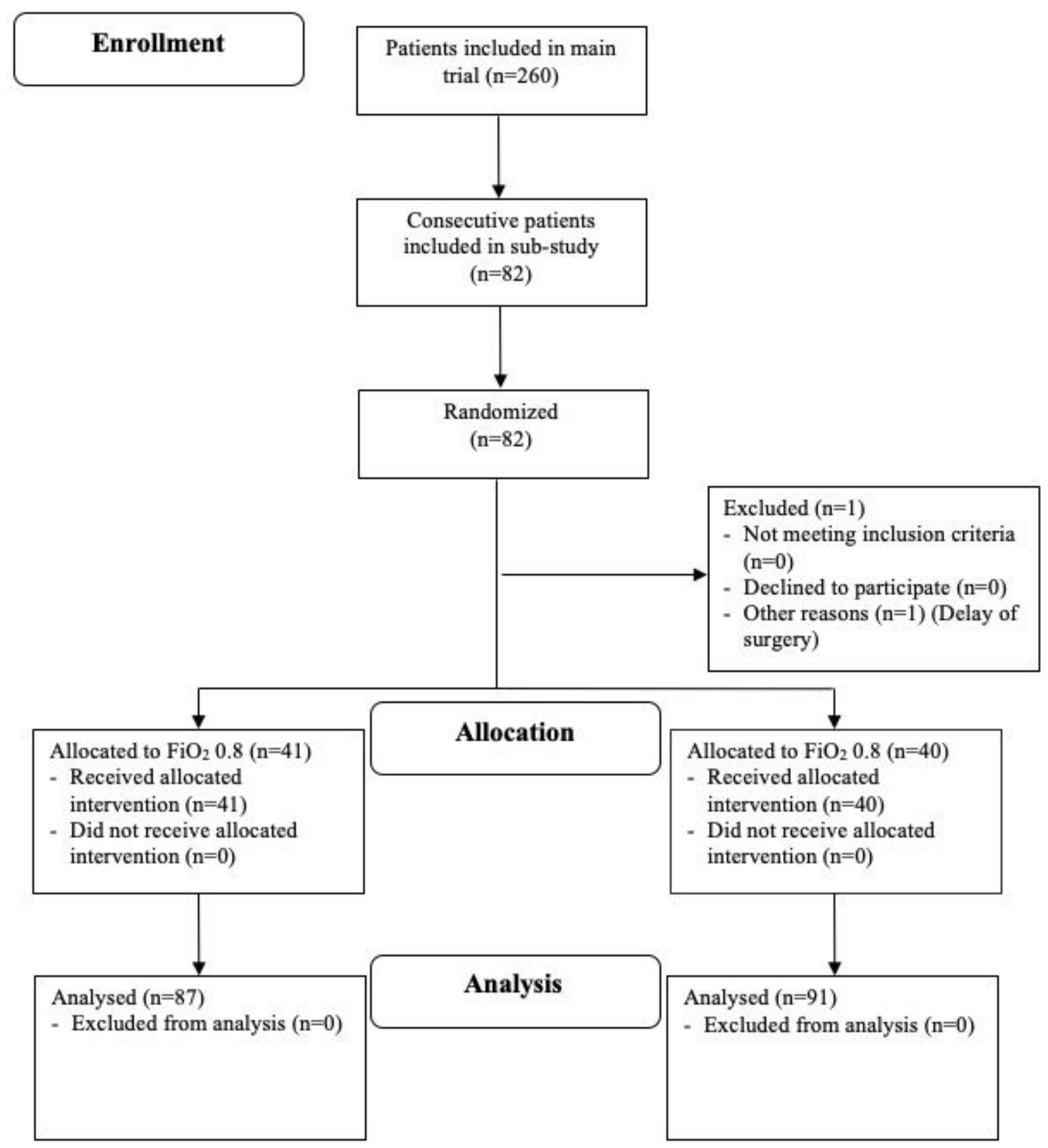

\section{Figure 1}

Patient Flow Diagram; Design and Form in Accordance with the 2010 CONSORT Guidelines.25 


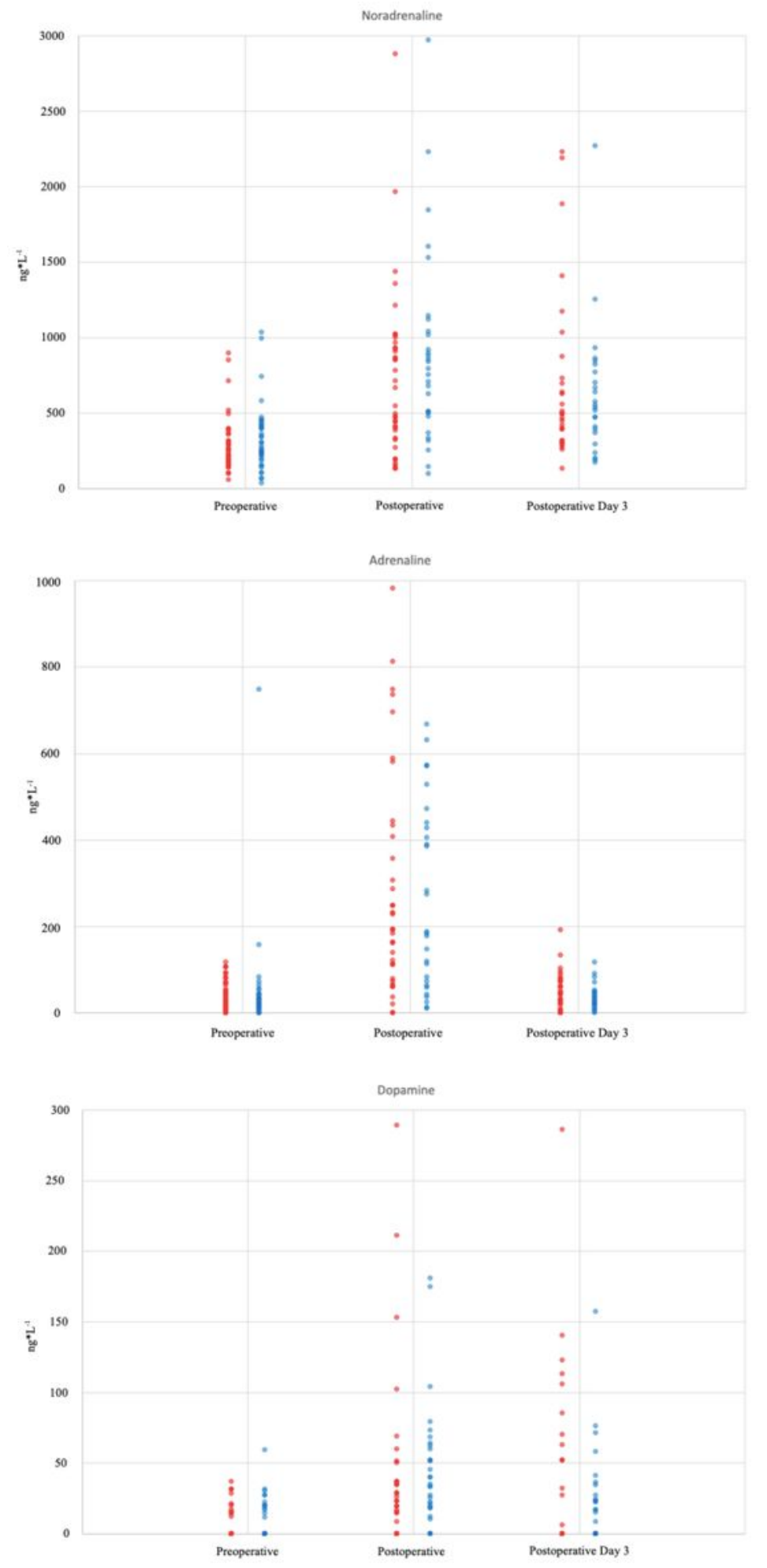

\section{Figure 2}

a. Noradrenaline b. Adrenaline c. Dopamine a-c. Plots showing the perioperative course of plasma a) noradrenaline $b$ ) adrenaline and $c$ ) dopamine concentrations between patients who received $80 \%$ fraction of inspired oxygen (blue) and patients who received 30\% fraction of inspired oxygen (red). Each circle represents one patient at each timepoint. 


\section{Supplementary Files}

This is a list of supplementary files associated with this preprint. Click to download.

- CONSORT2010ChecklistPerioperativeMedicine.doc 